\title{
Gelatinase-sensitive nanoparticles loaded with photosensitizer and STAT3 inhibitor for cancer photothermal therapy and immunotherapy
}

Lin-Lin Bu ${ }^{1,2^{*+}} \oplus$, Han-Qi Wang ${ }^{1+}$, Yuanwei Pan ${ }^{3}$, Lei Chen ${ }^{1}$, Hao Wu ${ }^{1}$, Xianjia Wu ${ }^{3}$, Chenchen Zhao ${ }^{3}$, Lang Rao ${ }^{3^{*}}$, Bing Liu, ${ }^{1,2^{*}}$ and Zhi-Jun Sun ${ }^{1,2^{*}}$

\begin{abstract}
Matrix metalloproteinase (MMP) 2 and 9 are the family members of proteases normally up-regulated in tumor to enhance the invasion and metastatic of tumor cells, and are associated with poor outcome of head and neck squamous cell carcinomas (HNSCCS). In the present work, MMPs-degradable gelatin nanoparticles (GNPs) are simultaneously loaded with photosensitizer indocyanine green (ICG) along with signal transducer activator of transcription 3 (STAT3) inhibitor NSC74859 (NSC, N) for efficient photothermal therapy (PTT) and immunotherapy of HNSCCs. In the tumor tissue, Gel-N-ICG nanoparticle was degraded and encapsulated ICG and NSC were effectively released. Under near-infrared (NIR) irradiation, the released ICG nanoparticles enabled effective photothermal destruction of tumors, and the STAT3 inhibitor NSC elicited potent antitumor immunity for enhanced cancer therapy. Based on two HNSCC mouse models, we demonstrated that Gel-N-ICG significantly delayed tumor growth without any appreciable body weight loss. Taken together, the strategy reported here may contribute that the stimuli-responsive proteases triggered nanoplatform could reduce tumor size more effectively in complex tumor microenvironment (TME) through combination of PTT and immunotherapy.
\end{abstract}

Keywords: Stimuli-responsive drug release, Indocyanine green, STAT3 inhibitor, Immunotherapy, Photothermal therapy

\footnotetext{
*Correspondence: lin-lin.bu@whu.edu.cn; Irao@szbl.ac.cn;

liubing9909@whu.edu.cn; sunzj@whu.edu.cn

†Lin-Lin Bu and Han-QiWang contributed equally to this work

${ }^{1}$ The State Key Laboratory Breeding Base of Basic Science of Stomatology

(Hubei-MOST) \& Key Laboratory of Oral Biomedicine Ministry

of Education, School \& Hospital of Stomatology, Wuhan University,

Wuhan 430079, China

${ }^{3}$ Institute of Biomedical Health Technology and Engineering, Shenzhen

Bay Laboratory, Shenzhen 518132, China

Full list of author information is available at the end of the article
}

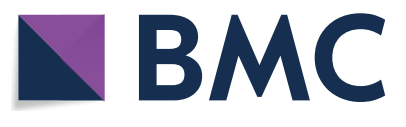
permits use, sharing, adaptation, distribution and reproduction in any medium or format, as long as you give appropriate credit to the original author(s) and the source, provide a link to the Creative Commons licence, and indicate if changes were made. The images or other third party material in this article are included in the article's Creative Commons licence, unless indicated otherwise in a credit line to the material. If material is not included in the article's Creative Commons licence and your intended use is not permitted by statutory regulation or exceeds the permitted use, you will need to obtain permission directly from the copyright holder. To view a copy of this licence, visit http://creativecommons.org/licenses/by/4.0/. The Creative Commons Public Domain Dedication waiver (http://creativeco mmons.org/publicdomain/zero/1.0/) applies to the data made available in this article, unless otherwise stated in a credit line to the data. 


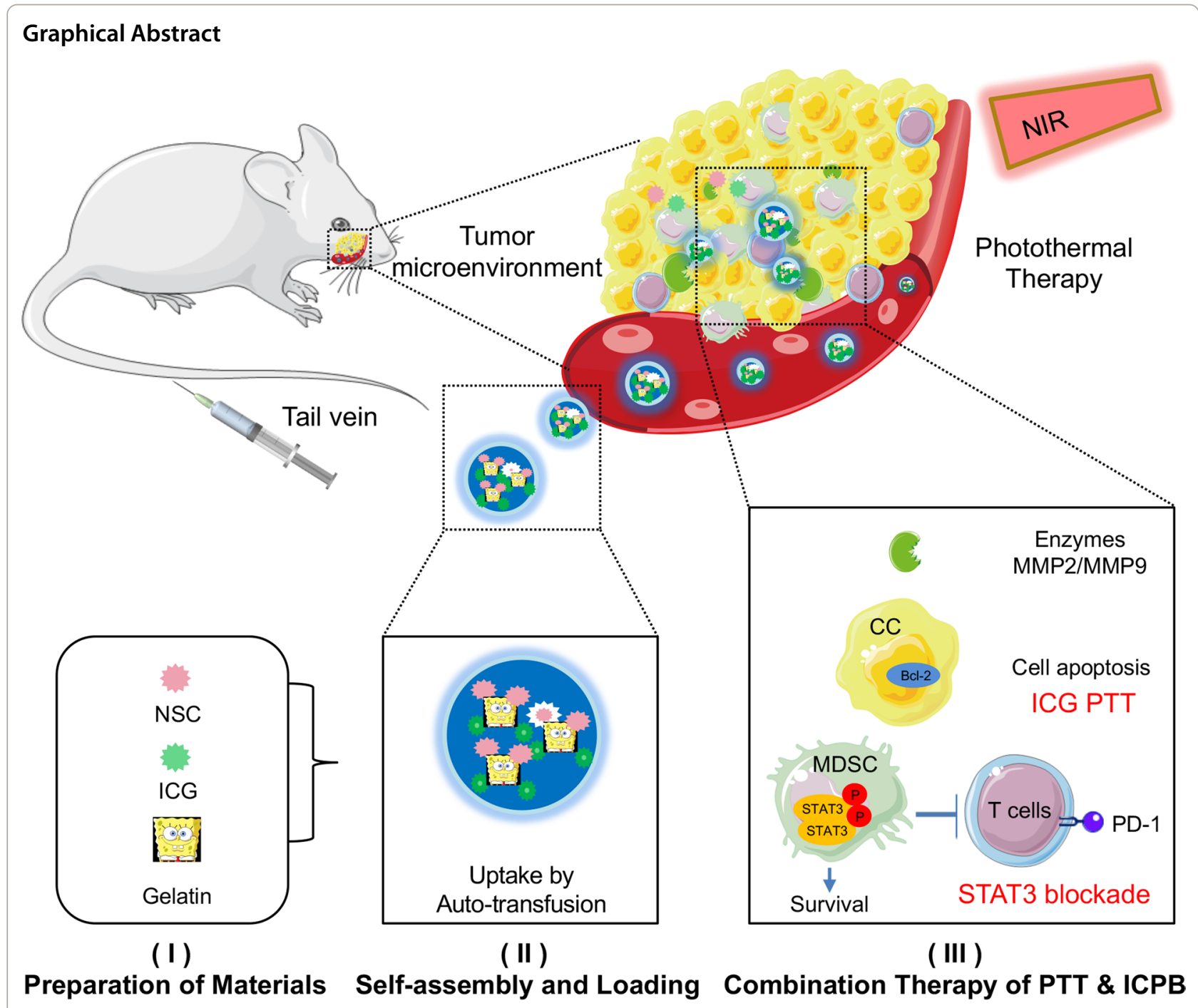

\section{Introduction}

As the sixth most common cancer around the world, there are more than 930,000 new patients of head and neck squamous cell carcinomas (HNSCCs) in 2020 , which account for about $90 \%$ of head and neck cancer patients [1, 2]. HNSCC is one of the most life-affecting malignant diseases, and except the deaths directly caused by HNSCC, the rate of suicide in HNSCC survivors (63.4 per 100,000 person-years) has been the second among all kinds of cancer [3]. Generally, current standard therapy of HNSCC still relies on surgery followed by conventional chemotherapy and radiotherapy $[4,5]$. However, due to the dysfunction caused by surgery and the chemotherapy resistance, the outcome of HNSCC treatment strategies is usually accompanied by cancer recurrence, metastasis and poor prognosis, and the overall survival rate in past three decades has improved modestly $[6,7]$. Nanotechnology demonstrated significant potential to reduce the side effects and increase drug targeting of immunotherapy [8]. So far, various nanomaterials have used in multiple fields including tumor treatment $[9,10]$. Among them, nanomaterial-based drug carriers such as liposomes [11, 12], polymer [13, 14], inorganic and hybrid protein-inorganic nanoparticles (NPs) [15-17] have been developed as nanocarriers to deliver various drugs, e.g., chemical anti-tumor drugs [18, 19], enzymes [20], antibody [21, 22], siRNA [23, 24], noble metal [25, $26]$ in order to improve the therapeutic effect.

Gelatin, the earliest proteinaceous material in formation of NPs and the carriage of drug through intravenous infusion, exhibits excellent characteristics such as biocompatibility, biodegradability, 
non-immunogenicity and safety in medical using, can be degraded by gelatinase and ease of bridge [27]. Among the various gelatinases that overexpressed in tumor microenvironment, matrix metalloproteinase (MMP) has been demonstrated to have a promising effect in enriching gelatin NPs [28, 29]. Previous studies demonstrated that gelatin-based NP can be used as MMP responsive carrier for tumor cell imaging in cancer treatment $[30,31]$. By loading with different drugs, gelatin NPs can play different role in inhibiting or diagnosing various cancers. For instance, Ruan S et al. designed a new way to improve targeting delivery efficiency and treatment outcome of glioma by loading gold NPs into gelatin NPs (GNPs) and decorating with doxorubicin (DOX) and Cy5.5 [32]. More recently, Chen et al. demonstrated the antitumor effect of GNPs encapsulating indocyanine green ICG and DOX in breast cancer [33].

As for drugs loaded in NPs, considering the immunosuppression and metastasis of cancer associated with the overexpression of programmed cell death ligand 1 (PD-L1) induced by constitutive activation of STAT3, NSC74859 (NSC, N), a signal transducer activator of transcription 3 (STAT3) inhibitor targeting Src Homology 2 (SH2) domain, was used to delay the growth of HNSCC in our previous works [34]. Moreover, indocyanine green (ICG), approved by FDA in clinical use, is a near-infrared dye and widely used in fluorescence imaging (FI) and photothermal therapy (PTT) [35, 36]. As the novel way in cancer treatment, principle of PTT is converting light to heat by exposing photosensitive nanoparticles to laser which can induce the tumor cell elimination and enhance the effect of immune agents via the transformation of the immunosuppressive "cold" tumor to the immunosensitive "hot" tumor [37, 38].

Herein, we developed a self-assemble gelatinase sensitive nanoparticle loaded with ICG and NSC (Gel-N-ICG) to achieve targeted drug delivery and HNSCC inhibition through both PTT and mufti-functional immunotherapy (Scheme 1). The supramolecular gelatin NPs (SGNPs) were prepared via a desolvation technique, ICG and STAT3 inhibitor NSC were co-immobilized into GNPs. Subsequently, the resulting Gel-N-ICG NPs accumulated in tumor site via the enhanced permeability and retention (EPR) effect after intravenous (i.v.) injecting into the donor mice [39]. When NPs arrived at targeting region, the drug delivery system were degraded by MMP with the release loaded drugs. The released ICG played the role in PTT as the photothermal adjuvant after the absorption in wavelength of $808 \mathrm{~nm}$. Meanwhile, released NSC played a role in immunotherapy through blocking the immune checkpoint protein and inducing the immune respond.

\section{Results and discussion}

Preparation and characterization of Gel-N-ICG nanoparticles

In this study, we synthesized the Gel-N-ICG NPs via desolvation technique according to previously studies, which has been demonstrated to be efficient in small nanoparticles formation [30, 40]. The drug loading was achieved by embedded the ICG and NSC into the hydrogel-like interior of SGNPs. The Gel-N-ICG NPs were prepared through self-assembly process on a microfluidic platform, the physiochemical parameters of Gel-N-ICG NPs including size, surface charge, stability can be controlled precisely and reproducibly by changing flow rates of fluids containing gelatin, ICG and NSC.

Transmission electron microscopy (TEM) was used to visualize the morphology of Gel-N-ICG NPs. As TEM image revealed, Gel-N-ICG NPs was about $100 \mathrm{~nm}$ with a spherical shape (Fig. 1a). Compared to SGNPs, the hydrodynamic diameters of Gel-N-ICG NPs measured by dynamic light scattering (DLS) were demonstrated to an increase due to the loading of NSC and ICG inside the SGNPs (Fig. 1b). Change in zeta potentials of SGNPs after loading with NSC and ICG was also presented in Fig. 1c. Moreover, in order to evaluate the stability of Gel-N-ICG NPs, the NPs were suspended in PBS buffer and DMEM containing $10 \% \mathrm{FBS}$ with different $\mathrm{pH}$ values from 2 to 10 and measured the size by DLS. As shown in Fig. 1d, DLS of Gel-N-ICG NPs in two different mediums with different $\mathrm{pH}$ had no significant difference which demonstrated the stability of these NPs. To confirm the successful loading of ICG into Gel and explore the ideal wavelength for PTT, Gel-N-ICG-NPs and free ICG were exposed into laser irradiation with various wavelength (Fig. 1e) and the results demonstrated that Gel-N-ICGNPs and free ICG all exhibited a characteristic absorption peak at about $808 \mathrm{~nm}$.

\section{In vitro and in vivo toxicity studies}

We then investigated the cytotoxicity of Gel and ICG in vitro. The cell viability of HNSCC cell line CAL27 and normal epithelial cell line HIOEC were monitored after treating with different concentrations of Gel or ICG. And the results did not present a significant change which demonstrated the safety of Gel and ICG (Additional file 1: Fig. S1). Moreover, the nanoparticle systems are of site-specific drug accumulation in tumors and have been reported as promising ways to overcome the potential in vivo toxicity of chemotherapy [41]. To test the potential toxicity of ICG and Gel-NPs in vivo, 15 ICR mice $(n=5)$ received an i.v. injection of $200 \mu \mathrm{L}$ PBS, or PBS containing ICG, Gel-NPs at the concentration of $5 \mathrm{mg}$ $\mathrm{mL}^{-1}$. After 30-day treatment, no death or significant 


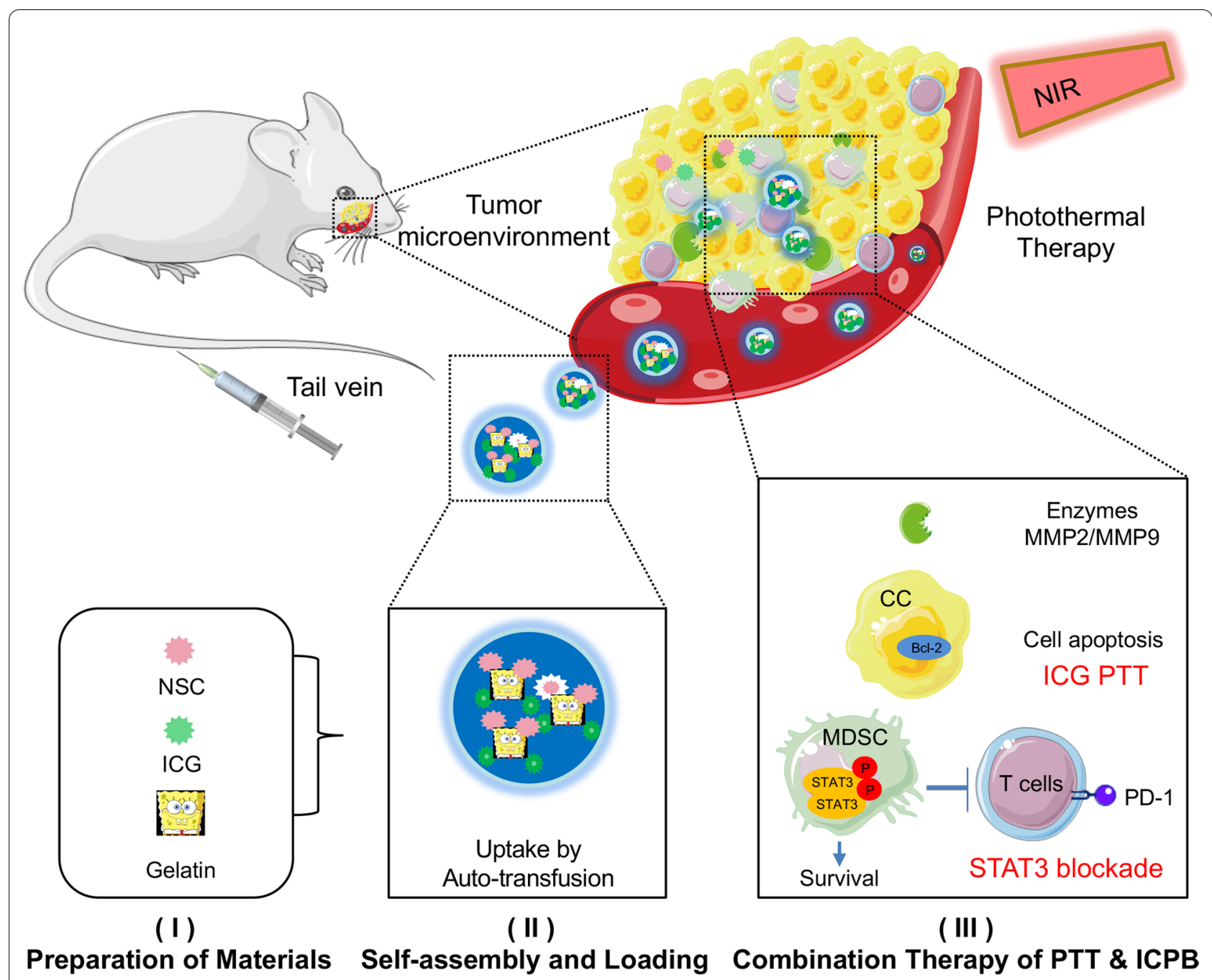

Scheme 1 I, II Preparation of ICG and NSC encapsulated supramolecular gelatin nanoparticles (Gel-N-ICG NPs) and uptake through i.v. injection; III schematic representation of PTT and multi-functional immunotherapy by Gel-N-ICG-NPs in the treatment of HNSCC

difference in body weight was observed among ICG and Gel-NPs groups compared to the control group (Additional file 1: Fig. S2), which demonstrated that treatment with ICG and Gel-NPs have no obviously overall side effects. Moreover, blood and major organs of all ICR mice including heart, liver, spleen, lung and kidney were collected on the 30th day for the blood biochemistry test and H\&E staining. Their blood parameters and H\&Estained slice images showed no significant differences between treated groups and control group (Additional file 1: Table S1, Fig. S3), which further demonstrated the safety of ICG and Gel-NPs application in vivo.

\section{Drug loading assay and enzyme-triggered release of Gel-N-ICG NPs}

To optimize the reaction conditions, the effect of drug loading and release in different weight ratio of NSC to
SGNPs and concentration of gelatinase were evaluated. To measure parameters of drug loading, SGNPs $(10 \mathrm{mg}$ $\mathrm{mL}^{-1}$ ) with weight ratio of NSC to SGNPs of 1:10, 2:10, $5: 10$, and 10:10, respectively, were quantified after swelling and loading of NSC into gelatin NPs for $24 \mathrm{~h}$. As presented in Fig. 1f, a higher drug loading efficiency (DLE) was achieved when the weight ratio of NSC to SGNPs was 1:10 while drug loading content (DLC) of NSC in Gel-N-ICG NPs had no significant difference between treated groups. And 1:10 was regarded as the optimized ratio of NSC to SGNPs with which the maximum absorption of NSC and SGNPs can be achieved. In the gelatinase solution, gelatin from encapsulated Gel-N-ICG NPs was degraded and allowed the release of NSC. As presented in Fig. 1g, in $\mathrm{pH}=7.4 \mathrm{PBS}$ buffer solutions, different concentrations of gelatinase effected the cumulative release of drug from Gel-N-ICG-NPs. It was demonstrated the 
a

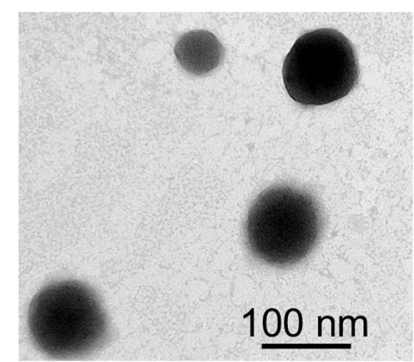

d

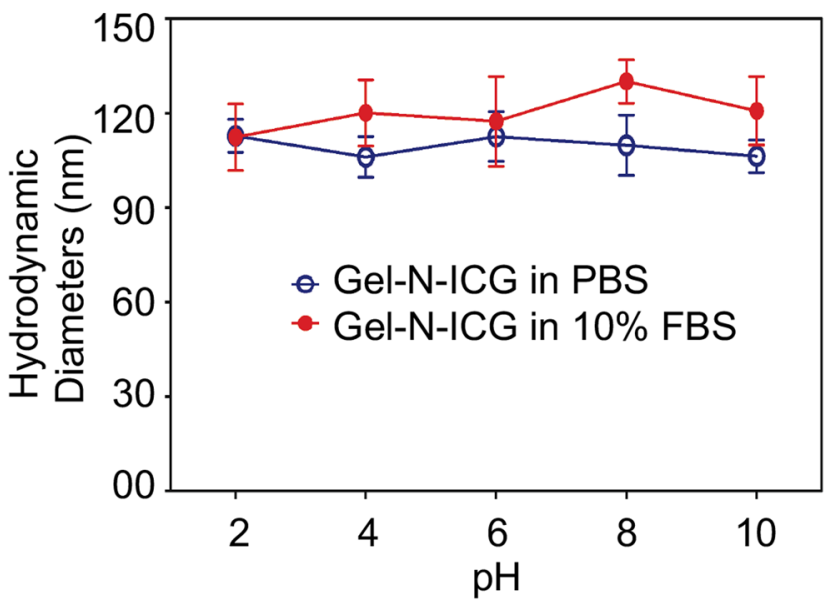

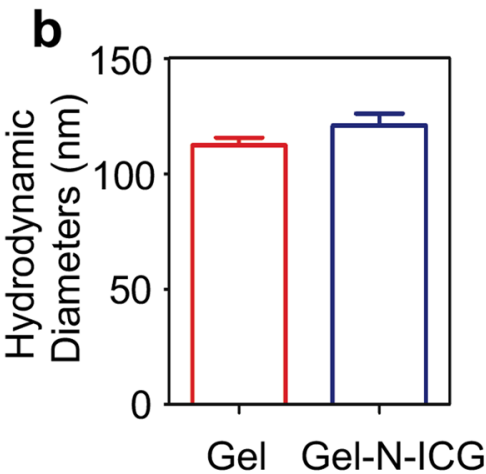

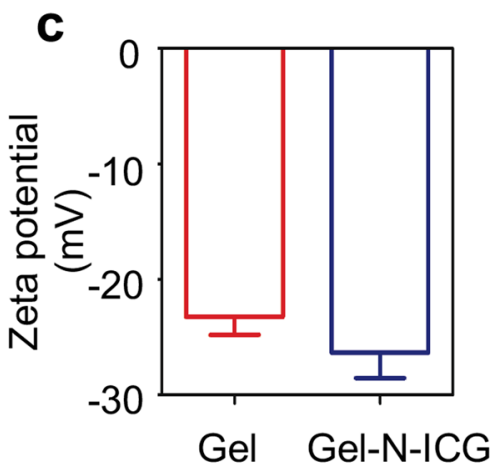

f

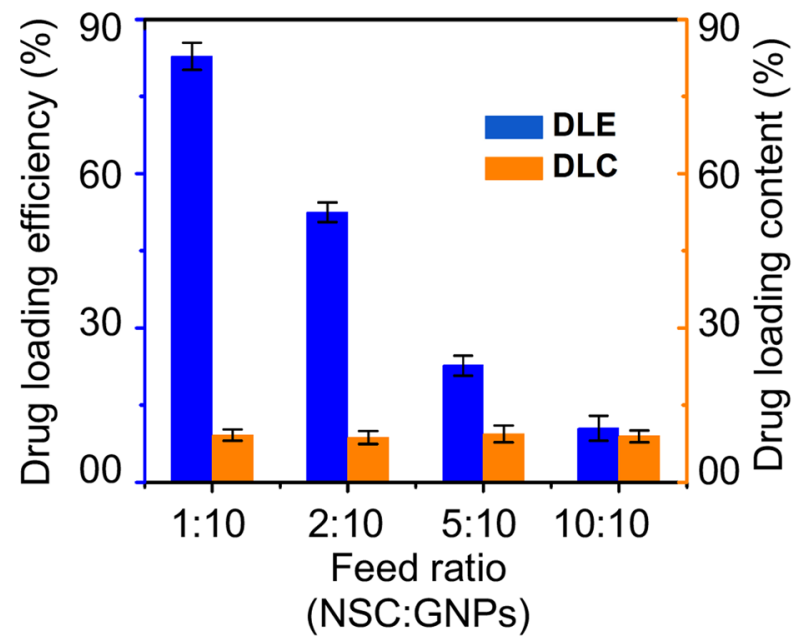

e

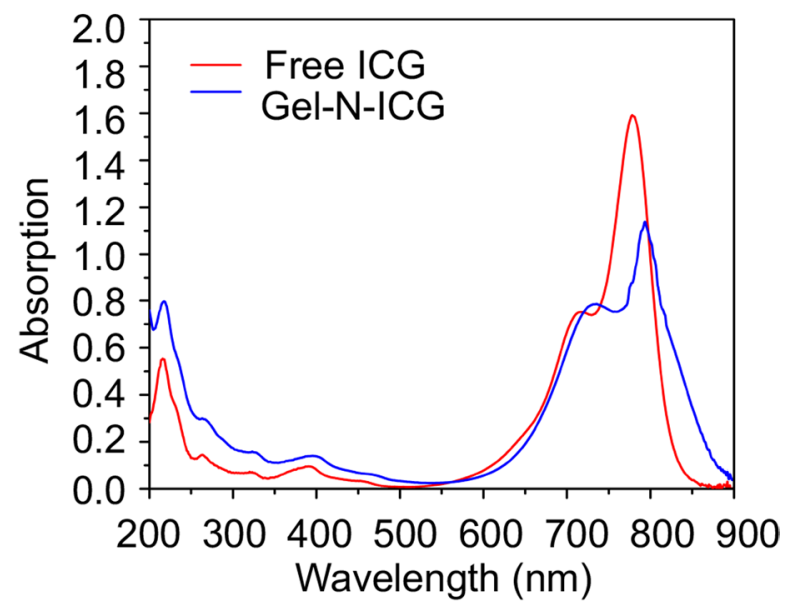

g

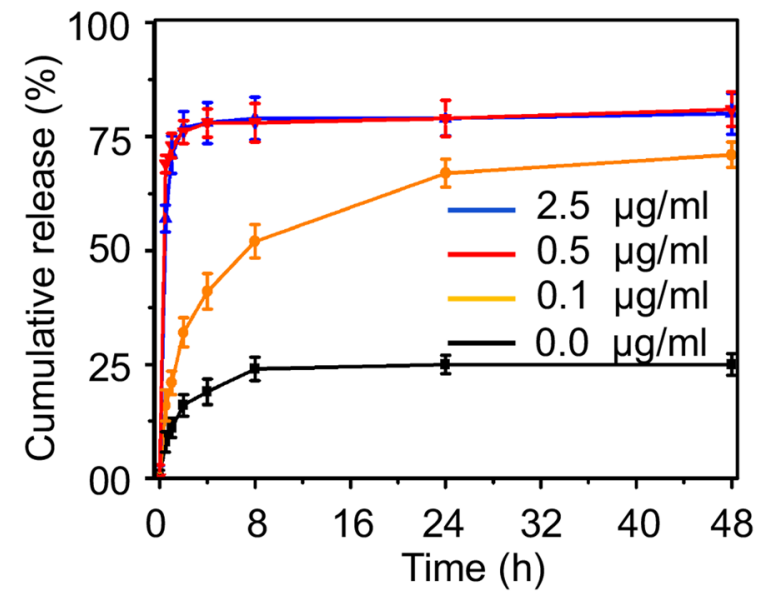

Fig. 1 Physicochemical characterization of Gel-N-ICG-NPs, loading efficiency, loading content, and release curve of NSC. a Representative TEM images of Gel-N-ICG-NPs. b Mean diameter size of bare Gel-NPs and Gel-N-ICG-NPs $(n=3$, mean \pm SD). $\mathbf{c}$ Zeta potential of Gel-NPs and Gel-N-ICG-NPs $(n=3$, mean \pm SD). $\mathbf{d}$ Stability studies of Gel-N-ICG-NPs under different $\mathrm{pH}$ values from 2 to 10 in PBS buffer and DMEM containing $10 \%$ FBS, respectively. e UV-vis-NIR spectra of Gel-N-ICG and free ICG which indicated the drug loading. $\mathbf{f}$ DLE and DLC of NSC in Gel-N-ICG-NPS. $\mathbf{g}$ Time course of drug release from Gel-N-ICG-NPs in different concentrations of gelatinase 
increase of total cumulative release with the increasing concentration of gelatinase (at the range of $0.0-0.5 \mu \mathrm{g} /$ $\mathrm{mL}$ ). And after $4 \mathrm{~h}$, the drug in 0.5 and $2.5 \mu \mathrm{g} / \mathrm{mL}$ gelatinase achieved a maximum release of more than $75 \%$.

\section{In vitro antitumor effect of Gel-N-ICG NPs}

Moving onward, we performed western blot and conducted a meta-analysis in TCGA data base to assess the protein expression of MMP9 and MMP2. The results displayed the overexpression of MMP9 and MMP2 in HNSCC cell line CAL27 compared to normal human epithelial cell line HIOEC (Fig. 2a, Additional file 1: Fig. S4), which will facilitate the degradation of gelatin NPs. Cell viability of CAL27 exhibited a remarkable regression as the increasing concentration of NSC which showed the anti-tumor efficacy of NSC. And there was no significant difference in cell viability between the treatment with NSC and Gel-N-ICG NPs without laser irradiation (Fig. 2b). To demonstrate the anti-tumor effect of immuno-photothermal therapy which combined Gel-NICG NPs with laser irradiation in HNSCC cells, CAL27 was treated with various solutions. Standard CCK-8 assay and Annexin V/PI staining were performed to show the apoptosis of CAL27 without any drug and after treatment with various solutions including Gel-ICG, Gel-N and Gel-N-ICG with laser irradiation for $24 \mathrm{~h}$ (Fig. 2c, d). As presented in flow cytometry analysis, cytotoxicity was mostly observed in Gel-N-ICG with laser irradiation treated group compared to other treated groups. And the apoptosis of CAL27 in vitro was significantly higher in treated groups compared to control group $(P<0.001)$.

\section{In vivo antitumor efficacy of Gel-N-ICG NPs in nude mice HNSCC model}

To evaluate the anti-tumor efficacy of Gel-N-ICG NPs in vivo, BALB/c nude mice bearing CAL27 tumor xenografts were treated with various solutions including PBS, ICG, Gel-ICG, and Gel-N-ICG with the exposure to an

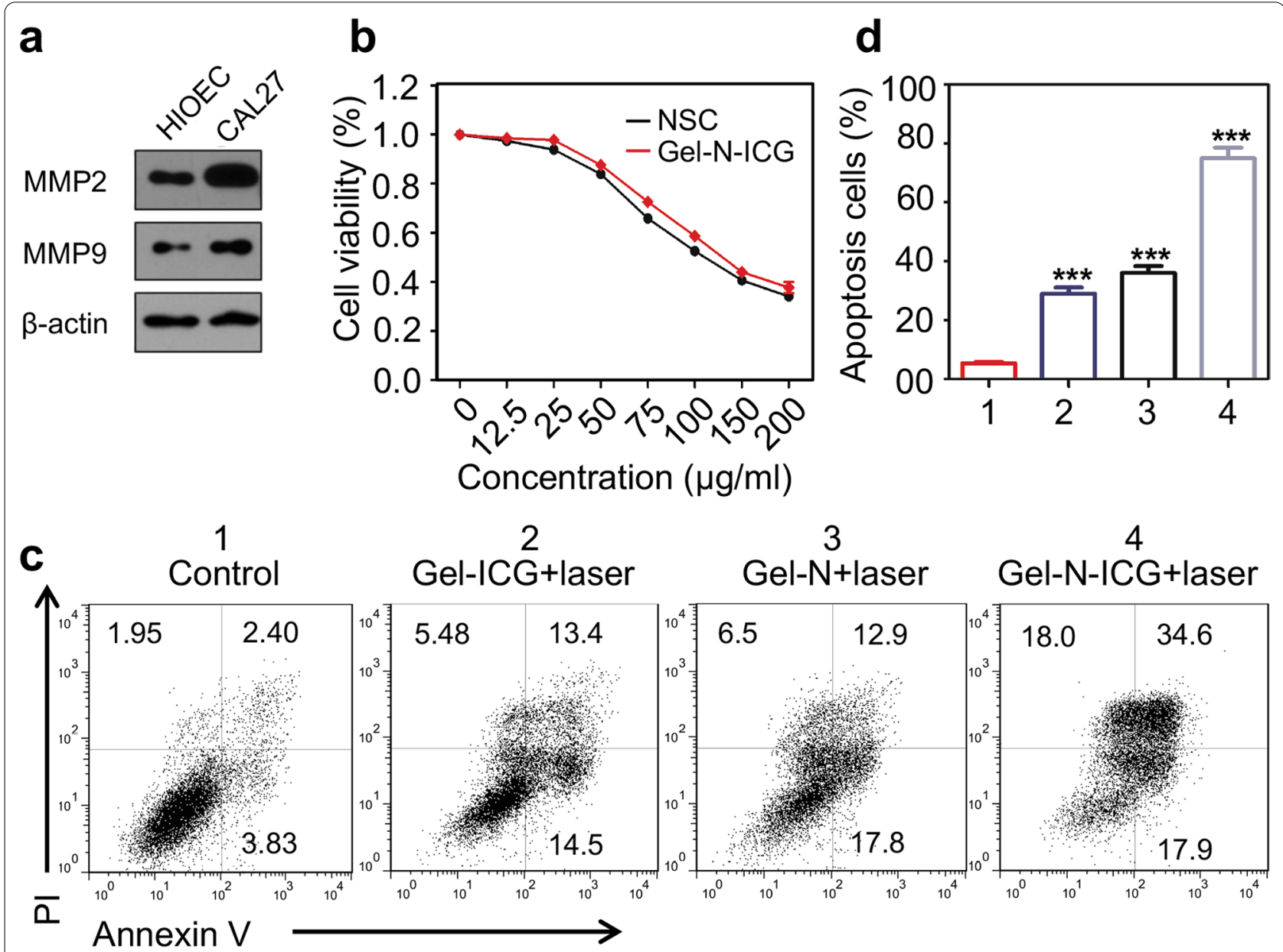

Fig. 2 Cell survivals of CAL27 cells after chemo-photothermal treatment. a Western blot qualitative in CAL27 and HIOEC. b Cell viability in different concentration of NSC and Gel-N-ICG NPs. c Flow cytometry analysis of CAL27 cells after different treatments. $\mathbf{d}$ Quantitative apoptosis cells (\%) in different treatment groups for $24 \mathrm{~h} .{ }^{*},{ }^{* *}$ and ${ }^{* * *}$ indicate $P<0.05, P<0.01$ and $P<0.001$, respectively, as compared with the PBS group 
$808 \mathrm{~nm}$ laser. The temperature of the tumor site in treated group gradually increased during the $5 \mathrm{~min}$ presence of laser irradiation which was attributed to the absorption of ICG at $808 \mathrm{~nm}$ laser, while no increase of temperature was observed in control group nearly (Fig. 3a). As images taken by infrared (IR) thermographic camera showed (Fig. 3c), the temperature increased to $54.4,56.2,58.3^{\circ} \mathrm{C}$, respectively, within $5 \mathrm{~min}$ laser irradiation in different treated groups. Furthermore, changes in tumor volume of nude mice models were regarded as the best direct index in evaluating the anti-tumor efficacy. As presented in Fig. 3b, tumor volume of each mouse was measured every other day from 14 day after various treatments. And on 28th day after treatment, all the tumor bearing nude mice were euthanized for the further experiments. Tumor volume in Gel-N-ICG with laser group achieved a remarkable control at 28th day after treatment, while tumor volumes in PBS, Gel-ICG, Gel-N, NSC, NSC-ICG with or without laser control groups presented a rapidly growth, which suggested that Gel-N-ICG NPs with laser can obtain the significant chemical and photothermal elimination of tumor compared to other groups (Fig. 3e, f). The body weights of mice at day 28 in Gel-N-ICG with laser group shown the least impacted compared to control group, which demonstrated the safety and antitumor effect, while in PBS with laser, ICG with laser and NSC groups, the body weights of mice showed a significantly regression (Fig. 3d).

\section{In vivo anti-tumor effect of Gel-N-ICG NPs in Tgfbr1/Pten 2cKO mice HNSCC model}

Bian et al. demonstrated that there were similar pathology and multiple molecular alterations of head and neck carcinogenesis between human and Tgfbr1/Pten double conditional knockout (2cKO) mice, so Tgfbr1/Pten 2cKO mouse was regarded as a suitable model in HNSCC tumorigenesis [42]. Here, we conducted the Tgfbr1/Pten $2 \mathrm{cKO}$ mice model to further evaluate the anti-tumor effect and immune response of Gel-N-ICG NPs in vivo. As HE stain confirmed (Additional file 1: Fig. S5a), tumor tissue of Tgfbr1/Pten 2cKO mice was similar to HNSCC tissue derived from human [42]. The expression level of gelatin enzyme including MMP2 was detected by immunohistochemically staining, as it presented in Additional file 1: Fig. S5b, MMP2 was overexpressed in Tgfbrl/ Pten 2cKO mice HNSCC compared to normal tissue. To evaluate the anti-tumor effect, Tgfbr1/Pten 2cKO mice received oral application of $2 \mathrm{mg}$ tamoxifen for consequent 5 days. On 30th day after induction, these Tgfbr1/ Pten 2cKO mice with squamous cell carcinoma growing in their oral cavity were treated with i.v. injection of various drugs with or without laser every other day for 15 days (Fig. 4a). As IR thermal images presented in Fig. 4b, the temperature of tumor site of Tgfbr1/Pten 2cKO mice raised from 33.7 to $63.7{ }^{\circ} \mathrm{C}$ in the group of Gel-NICG with $808 \mathrm{~nm}$ laser irradiation for $5 \mathrm{~min}$, which was higher than the $2.2^{\circ} \mathrm{C}$-increase in the group of PBS with laser irradiation. As presented in Fig. 4c, tumor volume in group 7 (Gel-N-ICG+ Laser) exhibited a significant reduction 15 days after application compared to tumor volume in group 1 (PBS) $(P<0.001)$. Moreover, there was no significant change in body weight of HNSCC mouse model between PBS control group and Gel-N-ICG group, which indicated that no systemic side effect was produced during the treatment combing PTT with immunotherapy (Fig. $4 \mathrm{~d}$ ). The representative tumor photo ex vivo before and after treatment (Fig. 4e) directly demonstrated that Gel-N-ICG NPs exposed into laser caused a remarkable tumor damage and inhibit the growth of tumor during the 2-week treatment.

\section{Immune response of Gel-N-ICG NPs in Tgfbr1/Pten 2cKO mice HNSCC model}

To explore the immune response of Gel-N-ICG NPs, Tgfbr1/Pten 2cKO mice were euthanized on 15th day after various treatments, and the tumor tissues, blood and spleen of mouse were harvested and analyzed through flow cytometry. $\mathrm{CD} 11 \mathrm{~b}^{+} \mathrm{Gr}^{+}$myeloid-derived suppressor cells (MDSCs) enhance the ability of tumor in immune evasion, invasion to the vasculature system and angiogenesis, which were associated with growth and metastasis of solid tumors [43]. As representative flow cytometry profiles shown in Fig. $5 \mathrm{a}-\mathrm{c}$, in Gel$\mathrm{N}$-ICG with laser group, the number of $\mathrm{CD}_{11 \mathrm{~b}^{+}} \mathrm{Gr}^{+}$ MDSCs in spleen, blood and tumor tissue were significantly decreased compared to PBS control group, which demonstrated the enhancement of anti-tumor immune response after treatment with Gel-N-ICG NPs exposed to laser irradiation. Moreover, the activation of immune response was further confirmed by flow cytometry (Fig. $5 \mathrm{~d}-\mathrm{f}$ ), the population of programmed cell death protein 1 (PD-1), the inhibitory receptor expressed on all $\mathrm{T}$ cells and limited protective immunity of $\mathrm{T}$ cells, presented a remarkable decrease in Gel-N-ICG NPs+ Laser group as well [44]. According to these results, Gel-NICG NPs with laser irradiation was demonstrated to have the ability to inhibit the immunosuppression of tumor microenvironment (TME), which can enhance the antitumor efficacy.

\section{Conclusions}

In summary, we have successfully created a gelatinase sensitive nanoparticle encapsulated ICG and STAT3 inhibitor (Gel-N-ICG) for efficient immunotherapy and PTT. Size-controllable SGNPs were easy to obtain. The Gel-N-ICG NPs performed good blood biochemistry, 


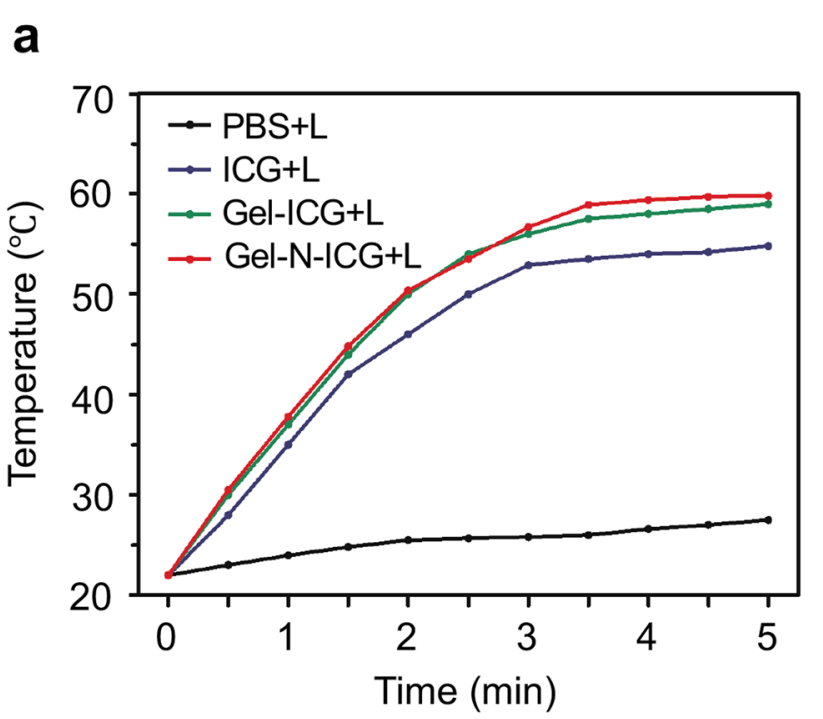

\section{b}
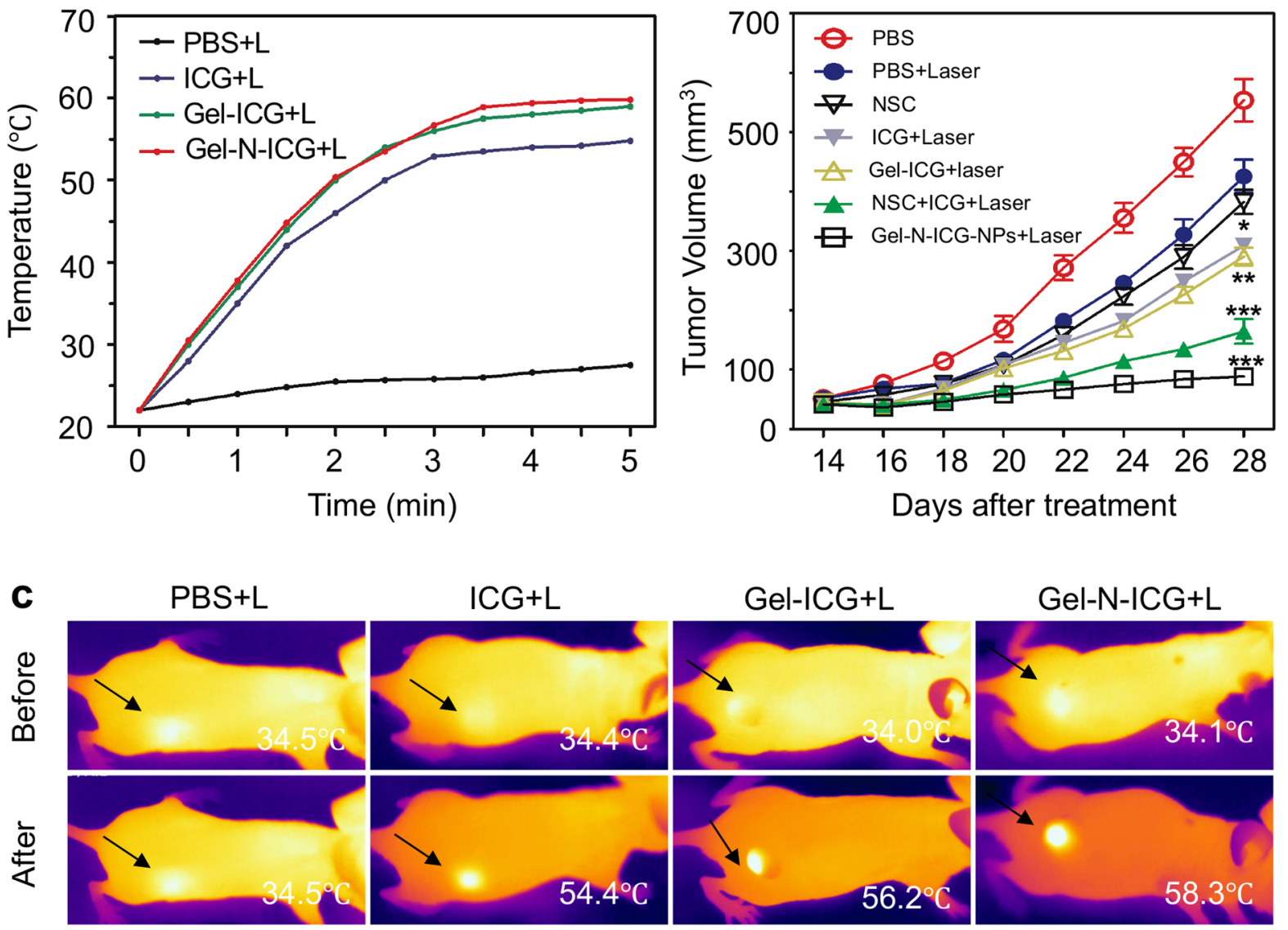

Low

High

d

e
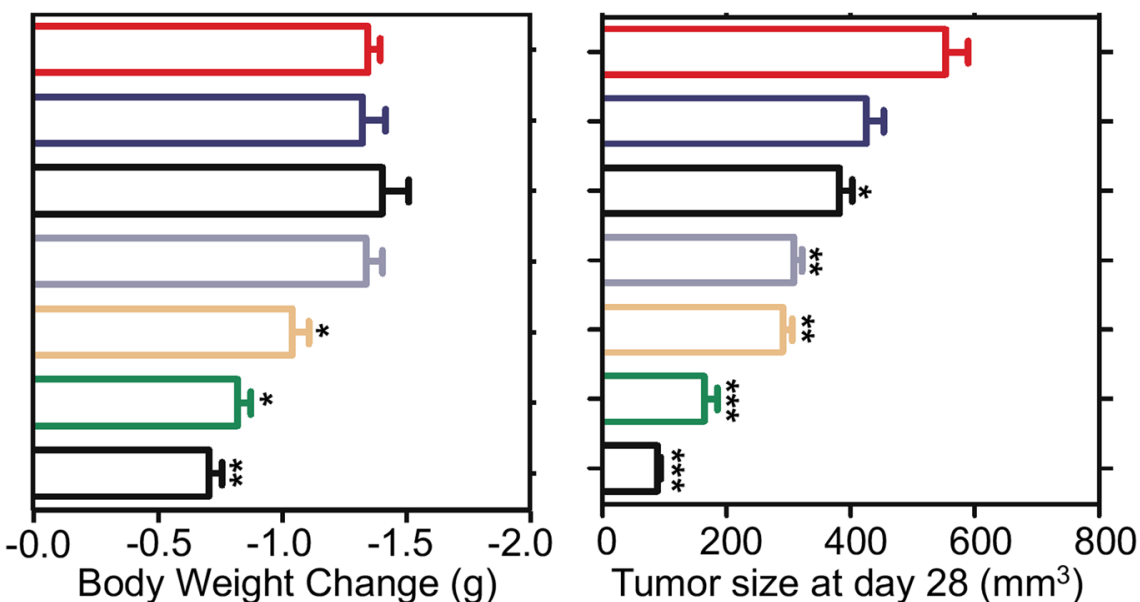

f

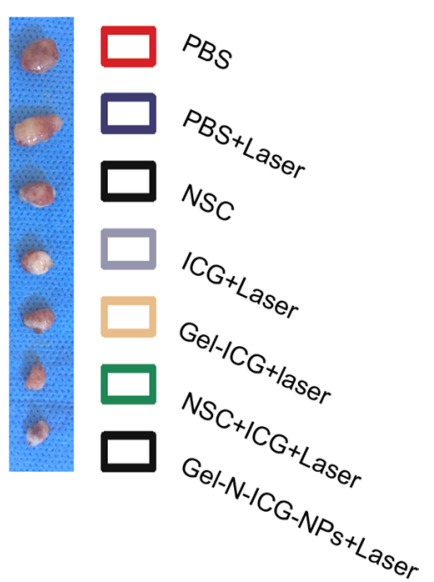

Fig. 3 Tumor therapy with Gel-N-ICG in xenograft mice model. a Maximum temperature profiles of the tumor site of nude mice after injection with different solutions with or without the $808 \mathrm{~nm}$ laser irradiation. b Tumor volume curves over 28 days after different treatment. c Representative in vivo IR thermal images of xenograft mice before and after injection with different solutions with a 5-min exposure to the laser irradiation. $\mathbf{d}$ Tumor bearing mice weight change, e tumor size at day 28 and $\mathbf{f}$ representative ex vivo tumor images from mice in different treatment groups. All data points represent as mean $\pm \mathrm{SD}(\mathrm{n}=5)$. NS, ${ }^{*}$, ${ }^{* *}$, and ${ }^{* * *}$ indicate no statistical difference, $P<0.05, P<0.01$, and $P<0.001$, respectively, as compared with the PBS group 


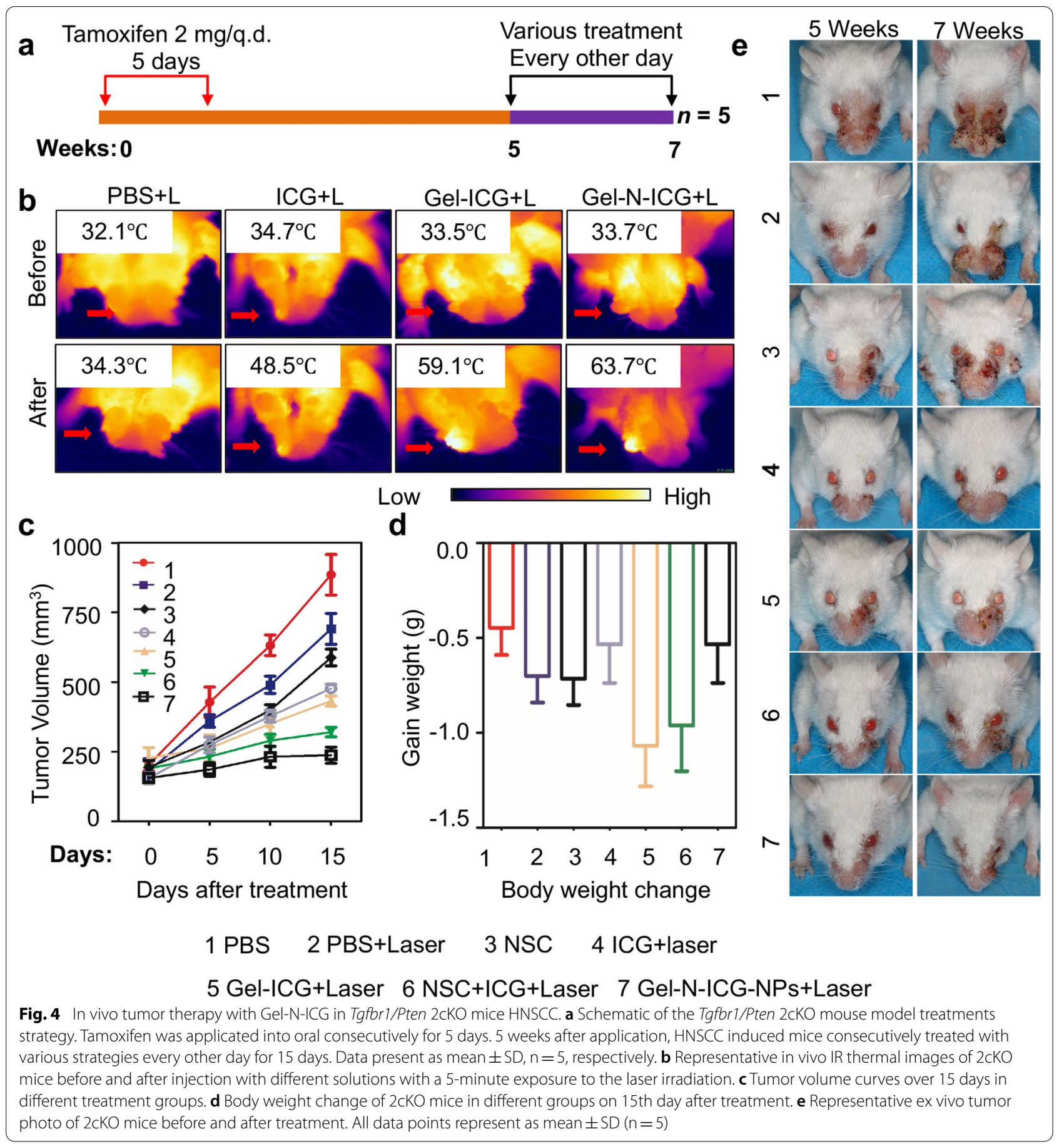

hematology tests, and histology analysis results which suggested no obvious toxicity occurred in the gelatinbased NPs in vitro and in vivo.

Meanwhile, the gelatin was degraded by MMP2/9, the gelatinase overexpressed in tumor microenvironment, and the encapsulated NSC and ICG were released in tumor site subsequently. Moreover, compared to the HNSCC xenograft nude mice model, the immune response, temperature of tumor surface after PTT and anti-tumor efficacy of Gel-N-ICG NPs were enhanced in Tgfbr1/Pten 2cKO head and neck carcinoma mice model which was of a closer TME to human HNSCC. 

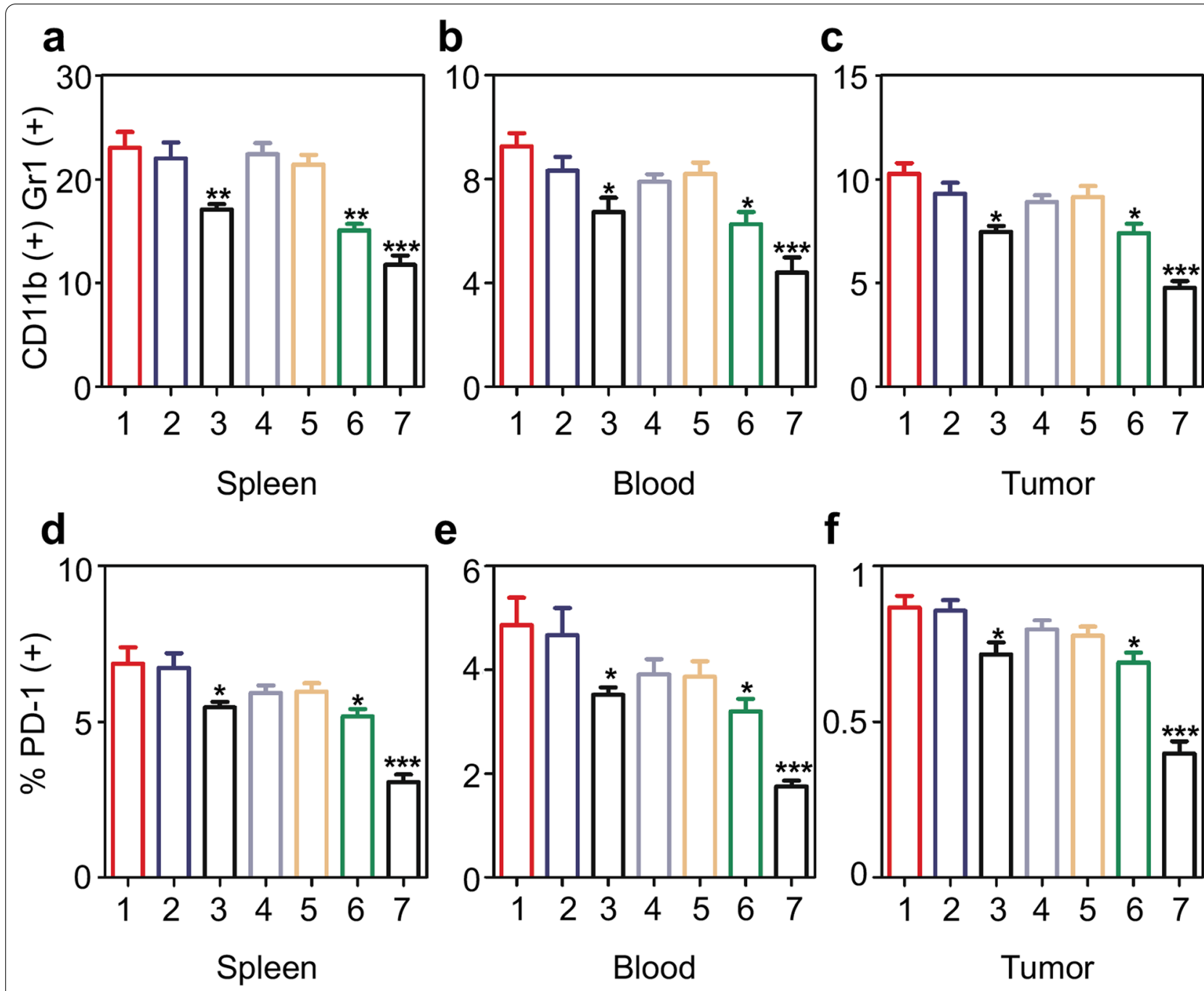

Tumor

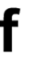

\section{PBS 2 PBS+Laser $3 \mathrm{NSC} \quad 4 \mathrm{ICG}+$ laser \\ 5 Gel-ICG+Laser 6 NSC+ICG+Laser 7 Gel-N-ICG-NPs+Laser}

Fig. 5 The population of MDSCs and PD-1 in Tgfbr1/Pten 2cKO mice. a-c Quantification of the percent of CD $11 b^{+} \mathrm{Gr}^{+}{ }^{+} \mathrm{MDSCs}$ in spleen, blood, tumor from mouse model in different treatment groups. $\mathbf{d}-\mathbf{f}$ Quantification of the percent of PD-1 ${ }^{+}$cells in spleen, blood, tumor from mouse model in different treatment groups. (Data presented as mean $\pm S D, n=5$ mice respectively. NS, ${ }^{*}{ }^{* *}$, and ${ }^{* * *}$ indicate no statistical difference, $P<0.05$,

$P<0.01, P<0.001$, as compared with the PBS group)

This approach has demonstrated an innovative antitumor delivery system for treating HNSCC. And this study may be proved helpful in reducing the solid tumor growth and increasing the efficacy of PTT and antitumor strategy.

\section{Methods}

\section{Cells, animal models and human HNSCC tissues}

CAL27 human squamous carcinoma cells were purchased and cultured as we reported before [34]. HIOEC (normal human epithelial cells) were used as a normal control. Two mouse models including ICR mice, BALB/c nude mice were purchased as previously reported and according to this previously study, we used CAL27 tumor cells to obtain the HNSCC xenografts [12]. And when the tumor size was at about $50 \mathrm{~mm}^{3}$, these xenograft mice were used for further studies. The inducible tissue-specific immunocompetent Tgfbr1/Pten 2cKO mice were obtained and maintained as previous described [42]. All the animal studies were approved by the Experimental Animal Ethics Committee of the School and Hospital of Stomatology at Wuhan University. Human HNSCC tissue with clinicopathological data and follow up was used for 
immunohistochemistry staining as previous study [45]. This study was approved by the Medical Ethics Committee of School and Hospital of Stomatology of Wuhan University and tumor tissues were derived from patients diagnosed with HNSCC.

\section{Materials and reagents}

Gelatin type B (225 bloom) from bovine skin, selenium (99\%, powder), sulfur (99.9\%, powder), glutaraldehyde solution (Grade I, 50\%) were obtained from Sigma Aldrich. Trioctylphosphine (TOP, 90\%) was obtained from Acros Organics. SU-8 2050 photoresist was purchased from MicroChem, USA. RTV615 Silicone Potting Compound was obtained from Momentive Performance Materials (Waterford, NY, USA). Recombinant human tissue inhibitor of metalloproteinases 2 (TIMP2) was obtained from Sino Biological (Beijing, China).

\section{Synthesis of Gel-N-ICG NPs, drug loading, release and nanoparticle characterization}

The SGNPs were prepared via desolvation method according to the previous studies [30]. The method to achieve the loading of NSC inside the gelatin NPs and the measure of the morphology, structure, ultravioletvisible-NIR absorbance spectra, dynamic diameters of nanoparticles, the encapsulation efficiency of NSC and ICG were referred to the previous literature [40]. Firstly, the NSC of which the concertation was $10 \mathrm{mg} \mathrm{mL}^{-1}$ with different weight ratio to SGNPs of 1:10, 2:10, 5:10, and 10:10, respectively, was added into synthesized SGNPs. Then, after a 24-h swell and loading at RT, the unloaded NPs were removed by dialysis (Spectra/Por 4, MWCO 12,000 to 14,000 ) against DI water overnight to purify NPs. After the dialysis of NPs, the concentration of free NSC and the DLE and DLC of NSC was measured via the mothed was described in previous literature, and to measure the release of NSC from Gel-N-ICG NPs in PBS buffer solutions with different concentration of gelatinase $(\mathrm{pH}=7.4)$, the HPLC was used to determine the Gel-NICG NPs release profiles as previous literature [40].

\section{Cell proliferation assay and Annexin V/PI staining}

To evaluate the cell proliferation, CCK8 assay and Annexin V/PI staining was conducted following the manufacture's instruction, and proliferation of cells were counted by flow cytometry.

\section{Dynamic light scattering (DLS)}

We used a Zetasizer Nano instrument (Zetasizer Nano ZS, Malvern Instruments Ltd., UK) with a 10-mW He-Ne laser and a thermoelectric temperature controller at the temperature of $25{ }^{\circ} \mathrm{C}$ and detection angle of $90^{\circ}$ to measure the hydrodynamic particle size. The data were processed subsequently by the Dispersion Technology Software (Malvern Instruments Ltd. UK).

\section{In vivo toxicity evaluation}

The potential in vivo toxicity of Gel and ICG was evaluated through i.v. injection of $200 \mu \mathrm{L}$ PBS, PBS containing ICG, PBS containing Gel with the concentration of $5 \mathrm{mg}$ $\mathrm{mL}^{-1}$ into $15 \mathrm{ICR}$ mice $(n=5)$, respectively. And every 3 days, we measured the body weights of treated mice. After the injection of solutions for 24 days, we euthanized the treated mice and collected their blood and major organs to measure the blood panel data and observed the organs sections stained with HE. The healthy mice were used as the control group.

\section{In vivo $\mathrm{PTT}$ evaluation}

35 mice bearing SCC $(n=5)$ received i.v. injection of 100 $\mu \mathrm{L}$ PBS or PBS with laser $\left(1 \mathrm{~W} \mathrm{~cm}{ }^{-2}, 5 \mathrm{~min}\right)$, NSC, ICG with laser, Gel-ICG with laser, NCS with ICG with laser and Gel-N-ICG NPs plus laser at the concentration of

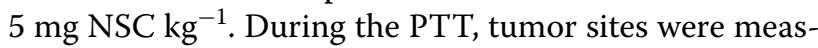
ured with an IR thermographic camera (FORTRIC225, Shanghai Thermal Image Electromechanical Technology Co. Ltd, China). After the PTT, tumor volumes of mice were measured every other day. On the 28th day after treatment, the changes of body weight of mice were measured and all mice were euthanized to collect the tumors. Then, the collected tumors were sectioned into $4 \mu \mathrm{m}$ and observed after staining using Ki-67.

Tgfbr1/Pten 2cKO mice were treated with a 5-day oral application of $2 \mathrm{mg}$ tamoxifen every day. On the 30th day after induction, SCCs were inducted in the mice oral cavity. To further compare the tumor of $2 \mathrm{cKO}$ mice with the human HNSCC, representative mice were euthanized to collect the tumor. Subsequently, total $352 \mathrm{cKO}$ mice $(n=5)$ received the i.v. injection of $100 \mu \mathrm{L}$ PBS or PBS with laser ( $\left.1 \mathrm{~W} \mathrm{~cm}^{-2}, 5 \mathrm{~min}\right)$, NSC, ICG with laser, Gel-ICG with laser, NCS with ICG with laser and GelN-ICG NPs plus laser at the concentration of $5 \mathrm{mg}$ NSC $\mathrm{kg}^{-1}$. During the PTT, IR thermographic camera was used to record the temperatures of the tumor. After the PTT, the tumor volumes of treated mice were measured every 5 days. At the 15th day after treatment, the changes of body weight were measured and all mice were euthanized to collect the tumor for the further experiments.

\section{Flow cytometry analysis}

Single cell suspensions from tumor site, spleens, and blood of Tgfbr1/Pten 2cKO mice with a variety of treatments were prepared. Tamoxifen inducted wide-type 
mice were set for flow cytometry analysis. And FITCconjugated anti-mouse CD11b antibody, PE-conjugated anti-mouse PD-1 and Gr-1 antibody were used to label these cells. FACS of these cells was conducted via flow cytometer.

\section{Statistical analyses}

Graph Pad Prism for Windows was used as introduced before for statistical analyses [12]. The difference among groups were detected by one-way ANOVA followed by the post-Tukey multiple comparison tests. Dates were represented as the mean \pm SD. Differences $(P<0.05)$ were of statistical significance.

\begin{abstract}
Abbreviations
MMP: Matrix metalloproteinase; HNSCCs: Head and necksquamous cell carcinomas; GNPs: Gelatin nanoparticles; ICG: Indocyanine green; NSC: NSC74859; PTT: Photothermal therapy; NIR: Near-infrared; DOX: Doxorubicin; FI: Fluorescence imaging; STAT3: Signal transduceractivator of transcription 3; SH2: Src homology 2; EPR: Enhanced permeabilityand retention; TEM: Transmission electronmicroscopy; DLS: Dynamic lightscattering; DLE: Drug loading efficiency; DLC: Drug loading content; IR: Infrared; TUNEL: Terminaldeoxynucleotidyl transferase-mediated deoxyuridinetriphosphate nick endlabeling; 2cKO: Double conditionalknockout; MDSCs: Myeloid-derivedsuppressor cells; PD-L1: Programmed cell deathligand 1; PD-1: Programmed cell deathprotein 1;TME: Tumor microenvironment; ATCC: American Type CultureCollection; CCK8: Cell Counting Kit-8.
\end{abstract}

\section{Supplementary Information}

The online version contains supplementary material available at https://doi. org/10.1186/s12951-021-01125-7.

Additional file 1: Table S1. Complete blood panel analysis of mice in the control and ICG, Gel-NPs.Standard deviations are based on 4 mice per group. WBC: white blood cell, RBC: red blood cell, HGB: hemoglobin, HCT: hematocrit, MCV: mean corpuscular volume, MCH: mean corpuscular hemoglobin, MCHC: mean corpuscular hemoglobin concentration, PLT: platelets, RDW: red blood cell distribution width. Figure S1. In vitrocytotoxicity of Gel and ICG against HNSCC cell line CAL27 and normal epithelialcell line HIOEC. (a, c) Cell viability of CAL27 cells with treatment with aseries of concentrations of Gel. (b, d) Cell viability of CAL27 cells withtreatment with a series of concentrations of ICG. Figure S2. Body weight change curves over a span of $30 \mathrm{~d}$ after the i.v.injection of PBS, ICG and Gel-NPs. FigureS3. Representative HE stained major organ slice images of mice $30 \mathrm{~d}$ after repeating i.v.injection of PBS, ICG and Gel-NPs; scalebars, $50 \mu \mathrm{m}$. Figure S4. Expression of Enzyme in Human HNSCC. (a, b) Meta-analysis of recent gene expression profiling for MMP2 and MMP9 by TCGA database $(n=566$, HNSCC $=522$, Normal $=44)$. $P<0.001$. (c) Over-expression of MMP2 in head and neck squamouscell carcinoma as compared with normal oral mucosa $(n=3)$; scale bars, 50 m. Figure $\mathbf{5 5}$. Gelatin enzyme expression in Tgfbr1/Pten 2cKO mice. (a) Representative HE staining of Tgfbr1/Pten 2cKO head and neck squamous cell carcinoma tissue $(n=3)$; scale bar, $50 \mu \mathrm{m}$. (b) Immunohistochemically staining indicate increase MMP2 expression in Tgfbr1/Pten 2cKO mice HNSCC $(n=3)$; scalebars, $50 \mu \mathrm{m}$.

\section{Acknowledgements}

Not applicable.

\section{Authors' contributions}

$\mathrm{LLB}$ and $\mathrm{HQW}$ contributed equally to this work. LR, BL and ZJS conceived the idea and designed the study. LLB, HQW, YP, LC, HW, XW and CZ performed the experiments. LLB analyzed the results and provided important technical supporting during the experiment. HQW wrote the manuscript. LLB, LR, BL and ZJS revised the final version of the manuscript. All authors read and approved the final manuscript.

\section{Funding}

This work was supported by Shenzhen Bay Laboratory Startup Fund (21310071), National Natural Science Foundation of China (81702703) and (81872203), China Postdoctoral Science Foundation (2018M630883, 2019T120688), Hubei Province Natural Science Foundation of China (2019CFB181). The authors declare no potential conflicts of interest with respect to the authorship and/or publication of this article.

\section{Availability of data and materials}

All data generated or analyzed during this study are included in this published article and the additional information.

\section{Declarations}

Ethics approval and consent to participate

All animal experiments were carried out under the guidelines evaluated and approved by the Experimental Animal Ethics Committee of the School and Hospital of Stomatology at Wuhan University (2017LUNSHENZIA34), and the study of human HNSCC tissue was approved by Medical Ethics Committee of School and Hospital of Stomatology of Wuhan University (2014LUNSHENZI06). Informed consent was obtained from all the patients.

\section{Consent for publication}

All authors of this study agreed to publish.

\section{Competing interests}

The authors declare no competing financial interests.

\section{Author details}

${ }^{1}$ The State Key Laboratory Breeding Base of Basic Science of Stomatology (Hubei-MOST) \& Key Laboratory of Oral Biomedicine Ministry of Education, School \& Hospital of Stomatology, Wuhan University, Wuhan 430079, China. ${ }^{2}$ Department of Oral \& Maxillofacial-Head \& Neck Oncology, School \& Hospital of Stomatology, Wuhan University, Wuhan 430079, China. ${ }^{3}$ Institute of Biomedical Health Technology and Engineering, Shenzhen Bay Laboratory, Shenzhen 518132, China.

Received: 2 September 2021 Accepted: 4 November 2021

Published online: 21 November 2021

\section{References}

1. Sung H, Ferlay J, Siegel RL, Laversanne M, Soerjomataram I, Jemal A, Bray F. Global cancer statistics 2020: GLOBOCAN estimates of incidence and mortality worldwide for 36 cancers in 185 countries. CA Cancer J Clin. 2021;71(3):209-49.

2. Chow LQM. Head and neck cancer. N Engl J Med. 2020;382(1):60-72.

3. Osazuwa-Peters N, Simpson MC, Zhao L, Boakye EA, Olomukoro SI, Deshields T, Loux TM, Varvares MA, Schootman M. Suicide risk among cancer survivors: head and neck versus other cancers. Cancer. 2018;124(20):4072-9.

4. Cramer JD, Burtness B, Ferris RL. Immunotherapy for head and neck cancer: recent advances and future directions. Oral Oncol. 2019;99:104460.

5. Budach W, Bölke E, Kammers K, Gerber PA, Orth K, Gripp S, Matuschek C. Induction chemotherapy followed by concurrent radio-chemotherapy versus concurrent radio-chemotherapy alone as treatment of locally advanced squamous cell carcinoma of the head and neck (HNSCC): a meta-analysis of randomized trials. Radiother Oncol. 2016;118(2):238-43.

6. Siegel RL, Miller KD, Jemal A. Cancer statistics, 2018. CA Cancer J Clin. 2018;68(1):7-30

7. Johnson DE, Burtness B, Leemans CR, Lui VWY, Bauman JE, Grandis JR. Head and neck squamous cell carcinoma. Nat Rev Dis Prim. 2020;6(1):92.

8. Cai L, Xu J, Yang Z, Tong R, Dong Z, Wang C, Leong KW. Engineered biomaterials for cancer immunotherapy. MedComm. 2020;1 (1):35-46. 
9. Teong SP, Zhang Y. Calcium carbide and its recent advances in biomass conversion. J Bioresour Bioprod. 2020;5(2):96-100.

10. Xia Z, Li J, Zhang J, Zhang X, Zheng X, Zhang J. Processing and valorization of cellulose, lignin and lignocellulose using ionic liquids. J Bioresour Bioprod. 2020;5(2):79.

11. Olusanya TO, Haj Ahmad RR, Ibegbu DM, Smith JR, Elkordy AA. Liposomal drug delivery systems and anticancer drugs. Molecules. 2018;23(4):907.

12. Rao L, Bu L-L, Meng Q-F, Cai B, Deng W-W, Li A, Li K, Guo S-S, Zhang W-F, Liu W, Sun Z-J, Zhao X-Z. Antitumor platelet-mimicking magnetic nanoparticles. Adv Funct Mater. 2017;27(9):1604774.

13. Tao W, Zeng X, Wu J, Zhu X, Yu X, Zhang X, Zhang J, Liu G, Mei L. Polydopamine-based surface modification of novel nanoparticle-aptamer bioconjugates for in vivo breast cancer targeting and enhanced therapeutic effects. Theranostics. 2016;6(4):470-84.

14. Sur S, Rathore A, Dave V, Reddy KR, Chouhan RS, Sadhu V. Recent developments in functionalized polymer nanoparticles for efficient drug delivery system. Nano-Struct Nano-Objects. 2019;20:100397.

15. Li Z, Barnes JC, Bosoy A, Stoddart JF, Zink JI. Mesoporous silica nanoparticles in biomedical applications. Chem Soc Rev. 2012;41 (7):2590-605.

16. Elzoghby AO, Hemasa AL, Freag MS. Hybrid protein-inorganic nanoparticles: from tumor-targeted drug delivery to cancer imaging. J Control Release. 2016;243:303-22.

17. Dong Z, Feng L, Hao Y, Chen M, Gao M, Chao Y, Zhao H, Zhu W, Liu J, Liang C, Zhang Q, Liu Z. Synthesis of hollow biomineralized CaCO(3)polydopamine nanoparticles for multimodal imaging-guided cancer photodynamic therapy with reduced skin photosensitivity. J Am Chem Soc. 2018;140(6):2165-78.

18. Zhao H, Feng H, Liu D, Liu J, Ji N, Chen F, Luo X, Zhou Y, Dan H, Zeng X, Li J, Sun C, Meng J, Ju X, Zhou M, Yang H, Li L, Liang X, Chu L, Jiang L, He Y, Chen Q. Self-assembling monomeric nucleoside molecular nanoparticles loaded with 5-FU enhancing therapeutic efficacy against oral cancer. ACS Nano. 2015;9(10):9638-51.

19. Wang F, Wang YC, Dou S, Xiong MH, Sun TM, Wang J. Doxorubicin-tethered responsive gold nanoparticles facilitate intracellular drug delivery for overcoming multidrug resistance in cancer cells. ACS Nano. 2011:5(5):3679-92.

20. Xie W, Deng WW, Zan M, Rao L, Yu GT, Zhu DM, Wu WT, Chen B, Ji LW, Chen L, Liu K, Guo SS, Huang HM, Zhang WF, Zhao X, Yuan Y, Dong W, Sun ZJ, Liu W. Cancer cell membrane camouflaged nanoparticles to realize starvation therapy together with checkpoint blockades for enhancing cancer therapy. ACS Nano. 2019;13(3):2849-57.

21. Han, X., S. Shen, Q. Fan, G. Chen, E. Archibong, G. Dotti, Z. Liu, Z. Gu, and C. Wang, Red blood cell-derived nanoerythrosome for antigen delivery with enhanced cancer immunotherapy. Sci Adv, 2019. 5(10): p. eaaw6870.

22. Chen Q, Wang C, Zhang X, Chen G, Hu Q, Li H, Wang J, Wen D, Zhang Y, Lu Y, Yang G, Jiang C, Wang J, Dotti G, Gu Z. In situ sprayed bioresponsive immunotherapeutic gel for post-surgical cancer treatment. Nat Nanotechnol. 2019;14(1):89-97.

23. Zhu X, Tao W, Liu D, Wu J, Guo Z, Ji X, Bharwani Z, Zhao L, Zhao X, Farokhzad OC, Shi J. Surface De-PEGylation controls nanoparticle-mediated siRNA delivery in vitro and in vivo. Theranostics. 2017;7(7):1990-2002.

24. Gao LY, Liu XY, Chen CJ, Wang JC, Feng Q, Yu MZ, Ma XF, Pei XW, Niu YJ, Qiu C, Pang WH, Zhang Q. Core-shell type lipid/rPAA-Chol polymer hybrid nanoparticles for in vivo siRNA delivery. Biomaterials. 2014;35(6):2066-78.

25. Chen WH, Yang CX, Qiu WX, Luo GF, Jia HZ, Lei Q, Wang XY, Liu G, Zhuo RX, Zhang XZ. Multifunctional theranostic nanoplatform for cancer combined therapy based on gold nanorods. Adv Healthc Mater. 2015;4(15):2247-59.

26. Yusuf A, Casey A. Surface modification of silver nanoparticle (AgNP) by liposomal encapsulation mitigates AgNP-induced inflammation. Toxicol In Vitro. 2019:61:104641

27. Hong S, Choi DW, Kim HN, Park CG, LeeW, Park HH. Protein-based nanoparticles as drug delivery systems. Pharmaceutics. 2020;12(7):604.

28. Xu X, Wang Y, Lauer-Fields JL, Fields GB, Steffensen B. Contributions of the MMP-2 collagen binding domain to gelatin cleavage. Substrate binding via the collagen binding domain is required for hydrolysis of gelatin but not short peptides. Matrix Biol. 2004;23(3):171-81.
29. Saito M, Kimoto M, Araki T, Shimada Y, Fujii R, Oofusa K, Hide M, Usui T, Yoshizato K. Proteome analysis of gelatin-bound urinary proteins from patients with bladder cancers. Eur Urol. 2005;48(5):865-71.

30. Xu JH, Gao FP, Liu XF, Zeng Q, Guo SS, Tang ZY, Zhao XZ, Wang H. Supramolecular gelatin nanoparticles as matrix metalloproteinase responsive cancer cell imaging probes. Chem Commun. 2013;49(40):4462-4.

31. Zou Z, He X, He D, Wang K, Qing Z, Yang X, Wen L, Xiong J, Li L, Cai L. Programmed packaging of mesoporous silica nanocarriers for matrix metalloprotease 2-triggered tumor targeting and release. Biomaterials. 2015;58:35-45.

32. Ruan $\mathrm{S}, \mathrm{He} \mathrm{Q}, \mathrm{Gao}$ H. Matrix metalloproteinase triggered size-shrinkable gelatin-gold fabricated nanoparticles for tumor microenvironment sensitive penetration and diagnosis of glioma. Nanoscale. 2015;7(21):9487-96.

33. Chen X, Zou J, Zhang K, Zhu J, Zhang Y, Zhu Z, Zheng H, Li F, Piao JG. Photothermal/matrix metalloproteinase-2 dual-responsive gelatin nanoparticles for breast cancer treatment. Acta Pharm Sin B. 2021;11(1):271-82.

34. Bu LL, Yu GT, Wu L, Mao L, Deng WW, Liu JF, Kulkarni AB, Zhang WF, Zhang L, Sun ZJ. STAT3 induces immunosuppression by upregulating PD-1/PD-L1 in HNSCC. J Dent Res. 2017;96(9):1027-34.

35. Chen $Q$, Liang C, Wang C, Liu Z. An imagable and photothermal "abraxanelike" nanodrug for combination cancer therapy to treat subcutaneous and metastatic breast tumors. Adv Mater. 2015;27(5):903-10.

36. Yang R, Hou M, Gao Y, Lu S, Zhang L, Xu Z, Li CM, Kang Y, Xue P. Biomineralization-inspired crystallization of manganese oxide on silk fibroin nanoparticles for in vivo MR/fluorescence imaging-assisted tri-modal therapy of cancer. Theranostics. 2019;9(21):6314-33.

37. Yang W, Guo W, Le W, Lv G, Zhang F, Shi L, Wang X, Wang J, Wang S, Chang J, Zhang B. Albumin-bioinspired Gd:CuS nanotheranostic agent for in vivo photoacoustic/magnetic resonance imaging-guided tumor-targeted photothermal therapy. ACS Nano. 2016;10(11):10245-57.

38. Ma Y, Zhang Y, Li X, Zhao Y, Li M, Jiang W, Tang X, Dou J, Lu L, Wang F, Wang Y. Near-infrared II phototherapy induces deep tissue immunogenic cell death and potentiates cancer immunotherapy. ACS Nano. 2019;13(10):11967-80.

39. Kalyane D, Raval N, Maheshwari R, Tambe V, Kalia K, Tekade RK. Employment of enhanced permeability and retention effect (EPR): nanoparticle-based precision tools for targeting of therapeutic and diagnostic agent in cancer. Mater Sci Eng C Mater Biol Appl. 2019;98:1252-76.

40. Li LL, Xu JH, Qi GB, Zhao X, Yu F, Wang H. Core-shell supramolecular gelatin nanoparticles for adaptive and "on-demand" antibiotic delivery. ACS Nano. 2014;8(5):4975-83.

41. Marcazzan, S., E.M. Varoni, E. Blanco, G. Lodi, and M. Ferrari, Nanomedicine, an emerging therapeutic strategy for oral cancer therapy. Oral Oncol, 2018. 76: p. $1-7$.

42. Bian Y, Hall B, Sun ZJ, Molinolo A, Chen W, Gutkind JS, Waes CV, Kulkarni AB, Loss of TGF- $\beta$ signaling and PTEN promotes head and neck squamous cell carcinoma through cellular senescence evasion and cancer-related inflammation. Oncogene. 2012;31 (28):3322-32.

43. Yang L, Edwards CM, Mundy GR. Gr-1+CD11 b+ myeloid-derived suppressor cells: formidable partners in tumor metastasis. J Bone Miner Res. 2010;25(8):1701-6.

44. Sharpe AH, Pauken KE. The diverse functions of the PD1 inhibitory pathway. Nat Rev Immunol. 2018;18(3):153-67.

45. Wu L, Deng W-W, Yu G-T, Mao L, Bu L-L, Ma S-R, Liu B, Zhang W-F, Sun Z-J. B7-H4 expression indicates poor prognosis of oral squamous cell carcinoma. Cancer Immunol Immunother. 2016;65(9):1035-45.

\section{Publisher's Note}

Springer Nature remains neutral with regard to jurisdictional claims in published maps and institutional affiliations. 